\title{
The independent role of prenatal and postnatal exposure to active and passive smoking on the development of early wheeze in children
}

\author{
C.I. Vardavas ${ }^{1,2}$, C. Hohmann ${ }^{3,4}$, E. Patelarou ${ }^{1,5}$, D. Martinez $z^{6,7,8}$, \\ A.J. Henderson ${ }^{9}$, R. Granell ${ }^{9}$, J. Sunyer ${ }^{6,7,8,10}$, M. Torrent ${ }^{6,11}$, M.P. Fantini ${ }^{12}$, \\ D. Gori' ${ }^{12}$, I. Annesi-Maesano ${ }^{13,14}$, R. Slama ${ }^{15}$, L. Duijts ${ }^{16,17,18}$, J.C. de Jongste ${ }^{16}$, \\ J.J. Aurrekoetxea ${ }^{19,20,21}$, M. Basterrechea ${ }^{7,20,21}$, E. Morales ${ }^{6,7,22}$, \\ F. Ballester ${ }^{7,23}$, M. Murcia ${ }^{7,23}$, C. Thijs ${ }^{24}$, M. Mommers ${ }^{24}$, C.E. Kuehni ${ }^{25}$, \\ E.A. Gaillard ${ }^{26}$, C. Tischer ${ }^{27}$, J. Heinrich ${ }^{27,28}$, C. Pizzii ${ }^{29}$, D. Zugna ${ }^{29}$, \\ U. Gehring ${ }^{30}$, A. Wijga ${ }^{31}$, L. Chatzi ${ }^{1}$, M. Vassilaki ${ }^{1}$, A. Bergström ${ }^{32}$, E. Eller $^{33}$,

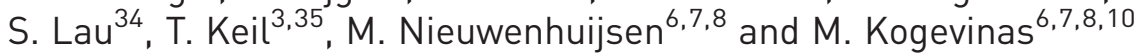

ABSTRACT Maternal smoking during pregnancy increases childhood asthma risk, but health effects in children of nonsmoking mothers passively exposed to tobacco smoke during pregnancy are unclear. We examined the association of maternal passive smoking during pregnancy and wheeze in children aged $\leqslant 2$ years.

Individual data of 27993 mother-child pairs from 15 European birth cohorts were combined in pooled analyses taking into consideration potential confounders.

Children with maternal exposure to passive smoking during pregnancy and no other smoking exposure were more likely to develop wheeze up to the age of 2 years (OR 1.11,95\% CI 1.03-1.20) compared with unexposed children. Risk of wheeze was further increased by children's postnatal passive smoke exposure in addition to their mothers' passive exposure during pregnancy (OR 1.29, 95\% CI 1.19-1.40) and highest in children with both sources of passive exposure and mothers who smoked actively during pregnancy (OR 1.73, 95\% CI 1.59-1.88). Risk of wheeze associated with tobacco smoke exposure was higher in children with an allergic versus nonallergic family history.

Maternal passive smoking exposure during pregnancy is an independent risk factor for wheeze in children up to the age of 2 years. Pregnant females should avoid active and passive exposure to tobacco smoke for the benefit of their children's health.

@ERSpublications

Prenatal and postnatal exposure to active and passive smoking independently increase early wheeze risk in children http://ow.ly/XZvQQ

Editorial comment in: Eur Respir J 2016; 48: 3-5.

This article has supplementary material available from erj.ersjournals.com

Received: June 262015 | Accepted after revision: Jan 272016 | First published online: March 102016

Support statement: Supported by the European Community's Seventh Framework Programme (FP7/2007-2013) under grant agreement no 226285 and, in part, by a Flight Attendant Medical Research Institute (FAMRI) grant (clinical investigator award 072058). The data collection and study teams of all participating birth cohorts were funded by local and/or national research organisations. The UK Medical Research Council and the Wellcome Trust (grant 092731) and the University of Bristol provide core support for ALSPAC study. Funding information for this article has been deposited with FundRef.

Conflict of interest: Disclosures can be found alongside this article at erj.ersjournals.com

Copyright OERS 2016 
Affiliations: ${ }^{1}$ Dept of Social Medicine, Faculty of Medicine, University of Crete, Heraklion, Greece. ${ }^{2}$ Center for Global Tobacco Control, Dept of Society, Human Development and Health, Harvard School of Public Health, Boston, MA, USA. ' Institute of Social Medicine, Epidemiology and Health Economics, Charité Universitätsmedizin Berlin, Berlin, Germany. ${ }^{4}$ University of Otago, Dunedin, New Zealand. ${ }^{5}$ Florence Nightingale Faculty of Nursing and Midwifery, King's College London, London, UK. ${ }^{6}$ Centre for Research in Environmental Epidemiology (CREAL), Barcelona, Spain. ${ }^{7}$ CIBER Epidemiología y Salud Pública (CIBERESP), Barcelona, Spain. ${ }^{8}$ Dept of Experimental and Health Sciences, University of Pompeu Fabra (UPF), Barcelona, Spain. ${ }^{9}$ School of Social and Community Medicine, University of Bristol, Bristol, UK. ${ }^{10} \mathrm{Hospital}$ del Mar Research Institute (IMIM), Barcelona, Spain. ${ }^{11}$ IB-Salut, Area de Salut de Menorca, Spain. ${ }^{12}$ Dept of Biomedical and Neuromotor Sciences, University of Bologna-Alma Mater Studiorum, Bologna, Italy. ${ }^{13}$ Inserm, Epidemiology of Allergic and Respiratory diseases (EPAR) Dept, U1136, Medical School SaintAntoine, Univ6, Sorbonne Universités Paris, Paris, France. ${ }^{14}$ UPMC, EPAR UMR-S 707, Medical School SaintAntoine, Univ6, Sorbonne Universités Paris, Paris, France. ${ }^{15}$ Team of Environmental Epidemiology applied to Reproduction and Respiratory Health, Inserm and Univ Grenoble Alpes Joint Research Centre (IAB, U823), Grenoble, France. ${ }^{16}$ The Generation R Study Group, Dept of Paediatrics, Division of Respiratory Medicine, Erasmus MC, University Medical Center, Rotterdam, The Netherlands. ${ }^{17}$ Dept of Paediatrics, Division of Neonatology, Erasmus MC, University Medical Center, Rotterdam, The Netherlands. ${ }^{18}$ Dept of Epidemiology, Erasmus MC, University Medical Center, Rotterdam, The Netherlands. ${ }^{19}$ Faculty of Medicine, University of the Basque Country (UPV/EHU), San Sebastian, Spain. ${ }^{20}$ BIODONOSTIA Health Research Institute, San Sebastian, Spain. ${ }^{21}$ Subdirección de Salud Pública de Gipuzkoa; Departamento de Sanidad del Gobierno Vasco, San Sebastian, Spain. ${ }^{22}$ Virgen de la Arrixaca Universtiy Hospital, IMIB-Arrixaca Research Institute, Murcia, Spain. ${ }^{23}$ FISABIO-Universitat de València-Universitat Jaume I Joint Research Unit of Epidemiology and Environmental Health, Valencia, Spain. ${ }^{24}$ Dept of Epidemiology, CAPHRI School of Public Health and Primary Care, Maastricht University, Maastricht, The Netherlands. ${ }^{25}$ Paediatric Respiratory Epidemiology, Institute of Social and Preventive Medicine, University of Bern, Switzerland. ${ }^{26}$ Institute for Lung Health, Dept of Infection Immunity and Inflammation, NIHR Leicester Respiratory Biomedical Research Unit, University of Leicester, Leicester, UK. ${ }^{27}$ Institute of Epidemiology I, German Research Centre for Environmental Health, Helmholtz Zentrum München, Neuherberg, Germany. ${ }^{28}$ Institute and Outpatient Clinic for Occupational, Social, and Environmental Medicine, Ludwig Maximilians University Munich, München, Germany. ${ }^{29}$ Cancer Epidemiology Unit, Dept of Medical Sciences, University of Turin, CPO Piedmont, Turin, Italy. ${ }^{30}$ Institute for Risk Assessment Sciences, Utrecht University, Utrecht, The Netherlands. ${ }^{31}$ Centre for Nutrition, Prevention and Health Services, National Institute for Public Health and the Environment (RIVM), Bilthoven, The Netherlands. ${ }^{32}$ Institute of Environmental Medicine, Karolinska Institutet, Stockholm, Sweden. ${ }^{33}$ Dept of Dermatology and Allergy Centre, Odense University Hospital, Odense, Denmark. ${ }^{34}$ Dept for Pediatric Pneumology and Immunology, Charité University Medical Centre, Berlin, Germany. ${ }^{35}$ Institute of Clinical Epidemiology and Biometry, University of Würzburg, Würzburg, Germany.

Correspondence: Manolis Kogevinas, Centre for Research in Environmental Epidemiology (CREAL), 88 Dr Aiguader Rd, Barcelona 08003, Spain. E-mail: kogevinasdacreal.cat

\section{Introduction}

It has been estimated that $40 \%$ of children worldwide breathe air polluted by tobacco smoke, with a detrimental impact on child health $[1,2]$. Exposure may start as early as in utero, due to maternal inhalation of other people's smoke and maternal active smoking, and may have an impact on multiple systems with possible lifelong implications $[2,3]$. The prenatal phase of life is a crucial period for human development during which maternal active or passive smoking may alter physiological mechanisms through various biochemical or metabolic pathways [4]. As lung development and maturation is a complex process from embryogenesis to early adolescence, it is possible that exposure to tobacco smoke during different periods may affect the developing lung in a different manner. Thus the association between the timing of each exposure and its impact on human health outcomes is an area of importance.

Wheezing is a common clinical sign of various infectious and noninfectious potentially life-threatening respiratory diseases in childhood, including asthma [5]. In epidemiological studies wheeze is considered a valid proxy measure for asthma, particularly at a very young age where an infant or preschool child with wheeze has not been seen and diagnosed with asthma by a doctor [6]. Preschool (viral) wheeze has been associated in several studies with the development of chronic obstructive pulmonary disease later in life, as well as with school-age asthma. Early childhood wheeze is a strong predictor for recurrent wheeze later in life, atopy, reduced lung function, airway responsiveness and doctor-diagnosed chronic asthma at school age [7-11].

As summarised by a recent meta-analysis of prospective cohort studies, exposure to tobacco smoke during pregnancy and early childhood increases the risk of developing wheeze [12]. However, to date there is limited evidence on the effects of maternal passive smoking during pregnancy and on the quantitative effect of different combinations of exposure to tobacco products (active smoking versus passive smoking of the mother) within different timeframes (prenatal versus postnatal) on the development of early wheezing. This lack of knowledge may be potentially attributable to the fact that very large cohort studies are needed to amass sufficient sample sizes for each potential time/exposure combination [13-15]. 
To address this research gap, our primary aim was to examine the association between maternal passive smoking during pregnancy and wheeze in children up to the age of 2 years. Furthermore, we assessed the independent and combined role of different sources of exposure during different timeframes (prenatal maternal passive smoking, prenatal maternal active smoking and children's postnatal passive smoking exposure) on the development of wheeze symptoms up to the age of 2 years in a pooled analysis and a meta-analysis of 15 prospective European mother-child cohorts.

\section{Methods}

Inclusion criteria and participating cohorts

The 15 cohorts providing primary data included in this analysis participated in the European project Environmental Health Risks in European Birth Cohorts (ENRIECO, www.enrieco.org) [16]. To be included in the analysis the cohorts had to recruit the newborns during pregnancy or shortly after birth, have information of maternal and infant exposure to active and passive smoking, have data available on the development of wheeze before the age of 2 years and data on major potential confounders. The list of included cohorts is given in online supplementary table S1. The cohorts commenced recruitment during 1990-2008 and each included between 330 and 14057 newborns (online supplementary table S1). A total of 37459 mother-child pairs were available from the birth cohorts, of which 27993 had complete data on second-hand smoke (SHS) exposure and wheeze (table 1). A comparison of subjects included and those excluded because of missing information on key passive smoking exposure or outcome variables is shown in online supplementary table S2. Multiple imputation was used to replace missing values in covariates (up to $9 \%$ for some covariates) and to address the potential bias and loss of precision that could result from incomplete case analyses. We created 10 imputations, generating 10 complete datasets that we analysed following the standard combination rules for multiple imputations [17]. The variables included as predictors were siblings, birthweight, gestational age, parental allergic status, parental education, sex of the child, damp and breastfeeding.

Definitions of exposure to active and passive smoking

Primary individual data were collected that included information on active maternal smoking and passive smoking obtained from questionnaire data submitted by each cohort. Responses were harmonised so as to

$\begin{array}{lc}\text { TABLE } 1 \text { Exposure, child and family characteristics of infants in the } 15 \text { European Union birth } \\ \text { cohorts } \\ \text { Prenatal maternal active smoking } \\ \quad \text { Not exposed } & 21685(77.5) \\ \text { Exposed } & 6308(22.5) \\ \text { Prenatal maternal passive smoking } & 9942(35.5) \\ \quad \text { Not exposed } & 18051(64.5) \\ \text { Exposed } & 16466(58.8) \\ \text { Postnatal child passive smoking } & 11527(41.2) \\ \text { Not exposed } & \\ \text { Exposed } & 9309(33.3) \\ \text { Health and demographic characteristics } & 13525(53.2) \\ \text { Any wheeze } & 2553 \\ \text { Parental allergy } & \\ \text { Missing data } & 14933(54.2) \\ \text { Parental education } & 10649(38.7) \\ \text { High } & 1957(7.1) \\ \text { Middle } & 454 \\ \text { Low } & \\ \text { Missing data } & 14457(51.7) \\ \text { Sex of the child } & 13534(48.4) \\ \text { Male } & 2 \\ \text { Female } & 3405 \pm 526 \\ \text { Missing data } & 349 \\ \text { Birthweight g } & 39.6 \pm 1.7 \\ \text { Missing data } & 258 \\ \text { Gestational age weeks } & \\ \text { Missing data } & \end{array}$

Data are presented as $n(\%), n$ or mean \pm SD. $n=27993$. Numbers of subjects with missing values for imputed variables are shown. 
follow four common exposure definitions, as follows. 1) Prenatal active smoking (maternal): maternal smoking at any time during pregnancy; 2) prenatal passive smoking (maternal): exposure of nonsmoking mothers to passive smoking during pregnancy (the father or other persons smoking in the household or workplace); 3 ) postnatal passive smoking (child): any exposure of the child to passive tobacco smoke from any source (maternal, paternal or other people smoking in proximity of the baby or within the house) at any time interval up to 2 years of age; 4) nonexposed: no active maternal smoking at any time during pregnancy, no prenatal maternal passive smoking and no postnatal child exposure to SHS.

Based on the different possible combinations of exposure to tobacco smoke, eight exclusive exposure groups were created and each participant was assigned only to one of the eight possible combinations of exposure, as depicted in table 2. This full detailed categorisation was available for a total of 27993 children.

\section{Definitions of outcomes and confounding factors}

Any wheezing during the first 2 years of life was the primary outcome variable assessed by parental self-report (yes/no) to the question whether the child has wheezed at any time in the last 12 months (6 months or since last assessment). The included birth cohorts used this (or a similarly phrased) question based on the widely used questionnaire from the International Study of Asthma and Allergies in Childhood (ISAAC), which validated the questions in many languages [18]. In three cohorts (CO.N.ER, LEICESTER 1990 and RHEA) information on wheezing was available only for the first year of life. Family history of atopy was defined as self-reported parental lifetime diagnosed asthma, allergic rhinitis and/or atopic eczema. Birth-related characteristics such as sex, birthweight and gestational age were collected for all participating cohorts together with information on siblings (yes/no). The socioeconomic status of the families was defined by the longest duration of school years spent by either mother or father and was categorised into three groups using cohort-specific tertiles as cut-offs: low, medium and high educational level [19]. Data on the existence of mould/dampness in the house, pet ownership, duration of breastfeeding and infant bedroom sharing were also collected but were not available for all cohorts.

\section{Ethics approval}

The included birth cohort studies were all approved by their local institutional review boards. All participants provided written informed consent for data collection and analysis.

\section{Statistical analysis}

We used a multilevel mixed-effects logistic regression to evaluate the effect of exposure to tobacco smoke on the development of child wheeze during the first 2 years of life, and calculated odds ratios and $95 \%$ confidence intervals. In the main model, adjustment was performed for sex of the child, family history of atopy (yes/no), parental education (high/middle/low), birthweight (g), gestational age (weeks) and siblings (yes/no). In addition, models without the inclusion of birthweight and gestational age were examined, since these factors may be influenced by prenatal exposure to SHS and could be intermediate variables on a pathway between exposure and outcome (wheeze). Cohort was introduced in all models as a random factor. Stratified analyses were performed for sex, parental atopy and geographical location of the cohorts (northern versus southern European). For analyses evaluating effects by region or cohort we examined exposure in three categories: nonexposed (nonsmoking mothers, mothers/infants not exposed to passive smoking); passive smokers only (nonsmoking mothers, mothers/infants exposed to SHS); and actively smoking mothers. A meta-analysis was performed to take into account the heterogeneity between the cohorts. A random effects model was used and a heterogeneity test based on the Q-statistic was performed considering a p-value $<0.10$ as statistically significant. Heterogeneity was further examined by omitting sequentially each cohort from the meta-risk estimate. Additional meta-analyses of cohort-specific estimates were performed with cohort-specific adjustment for variables that have been previously associated with wheeze but that were not all available for all cohorts (for example existence of mould/dampness in the house, pet ownership, duration of breastfeeding and infant bedroom sharing) [20-24]. Analyses were performed using the statistical package Stata 12.0 (StataCorp, College Station, TX, USA).

\section{Results}

Exposure to active and passive smoking and potential confounders

The prevalence of prenatal maternal active smoking at any time during pregnancy was $22.5 \%$, prenatal maternal passive smoking was $64.5 \%$ and children's postnatal passive smoking was $41.2 \%$ (table 1). Only $27.1 \%$ of children had no exposure to active or passive smoking during the prenatal or postnatal period, while $16.5 \%$ reported being exposed to prenatal maternal active and passive smoking and postnatal passive smoke exposure. Exposure to passive smoking during pregnancy was associated with exposure in the postnatal period. Specifically, among children of nonsmoking females who were unexposed to passive smoke during the gestational period, $83.4 \%$ continued to be unexposed during the postnatal period. 
TABLE 2 The role of different combinations of timeframes of exposure to active and passive smoking on the development of wheeze before the age of 2 years among 15 European birth cohorts

$\begin{array}{cccccc}\text { Cases/noncases }^{\#} & \begin{array}{c}\text { Prenatal maternal } \\ \text { active smoking }\end{array} & \begin{array}{c}\text { Prenatal maternal } \\ \text { passive smoking }\end{array} & \begin{array}{c}\text { Postnatal infant } \\ \text { passive smoking }\end{array} & \begin{array}{c}\text { Unadjusted OR } \\ (95 \% \mathrm{CI})^{\pi}\end{array} & \begin{array}{c}\text { Adjusted OR } \\ \left(95 \% \mathrm{CI}^{+}\right.\end{array}\end{array}$

\begin{tabular}{|c|c|c|c|c|c|c|}
\hline \multicolumn{7}{|l|}{$\begin{array}{l}\text { Nonsmoking mothers, mothers/infants } \\
\text { not exposed to passive smoking }\end{array}$} \\
\hline \multicolumn{7}{|l|}{$\begin{array}{l}\text { Nonsmoking mothers, } \\
\text { mothers/infants exposed to SHS }\end{array}$} \\
\hline 2) Prenatal passive smoking & $2531 / 5217$ & No & Yes & No & $1.03(0.95-1.11)$ & $1.11(1.03-1.20)$ \\
\hline 3) Postnatal passive smoking & 448/1055 & No & No & Yes & $1.03(0.90-1.18)$ & $1.06(0.93-1.20)$ \\
\hline $\begin{array}{l}\text { 4) Prenatal passive smoking + postnatal } \\
\text { passive smoking }\end{array}$ & $1613 / 3246$ & No & Yes & Yes & $1.19(1.09-1.30)$ & $1.29(1.19-1.40)$ \\
\hline \multicolumn{7}{|l|}{ Active smoking mothers" } \\
\hline $\begin{array}{l}\text { 5) Prenatal active smoking, no prenatal } \\
\text { or postnatal passive smoking }\end{array}$ & $100 / 214$ & Yes & No & No & $1.16(0.90-1.51)$ & $1.33(1.03-1.71)$ \\
\hline $\begin{array}{l}\text { 6) Prenatal active smoking + prenatal } \\
\text { passive smoking }\end{array}$ & $329 / 500$ & Yes & Yes & No & $1.38(1.16-1.65)$ & $1.51(1.29-1.77)$ \\
\hline $\begin{array}{l}\text { 7) Prenatal active smoking + postnatal } \\
\text { passive smoking }\end{array}$ & $217 / 333$ & Yes & No & Yes & $1.56(1.27-1.91)$ & $1.74(1.45-2.09)$ \\
\hline $\begin{array}{l}\text { 8) Prenatal active smoking + prenatal } \\
\text { passive smoking + postnatal passive smoking }\end{array}$ & $1876 / 2739$ & Yes & Yes & Yes & $1.63(1.50-1.78)$ & $1.73(1.59-1.88)$ \\
\hline
\end{tabular}

Data are presented as $\mathrm{n}$, unless otherwise stated. Subjects with complete information on exposures and outcomes $\mathrm{n}=27993$. SHS: second-hand smoke.\#: each category is exclusive: participants were categorised into one exposure combination only; ${ }^{\uparrow}$ : mixed-effects logistic regression including a random intercept for cohort; ${ }^{+}$: mixed-effects logistic regression including a random intercept for cohort adjusted for sex of the child, socioeconomic status, siblings, birthweight, parental atopic status and gestational age. Complete information on confounders was available for 23848 subjects and imputed for the remaining subjects. 
This percentage was lower $(36.4 \%)$ for children whose mothers were nonsmoking females but who had been exposed to passive smoking during pregnancy.

\section{Effect of exposure to maternal passive smoking during pregnancy}

In a detailed analysis of the eight mutually exclusive exposure categories (table 2) in which children never exposed to passive smoking were considered as a reference, increased risks of wheeze among children of nonsmoking mothers were observed for exposure only to maternal passive smoking during pregnancy (OR 1.11, 95\% CI 1.03-1.20). Exposure of the child to only postnatal passive smoking was associated with a small increase in risk (OR 1.06, 95\% CI 0.93-1.20). A higher risk was observed among children exposed to postnatal passive smoking in addition to maternal passive smoking during pregnancy (OR 1.29, 95\% CI 1.19-1.40) (table 2).

Estimates for models not adjusting for birthweight and gestational age were very similar (data not shown). Adjustment for several confoudners tended to increase the odds ratios compared to unadjusted estimates (table 2).

\section{Effect of additional exposure to maternal active smoking during pregnancy}

A higher risk of wheeze (table 2) was seen in children exposed to prenatal maternal active smoking and prenatal maternal passive smoking, but not to passive smoking after birth (OR 1.51, 95\% CI 1.29-1.77). Children exposed to prenatal maternal active smoking and postnatal passive smoking had an odds ratio of wheeze of 1.74 (95\% CI 1.44-2.09). Children exposed to prenatal maternal active smoking, prenatal maternal passive smoking and postnatal passive smoking had the highest likelihood of developing wheeze up to the age of 2 years (OR 1.73, 95\% CI 1.59-1.88). In each case, exposed children are compared with the same reference group of children never exposed to passive smoking.

\section{Effect modification by parental history of allergy and sex}

The odds ratio for the association between wheeze and exposure to passive smoking (prenatal passive smoking in nonsmoking mothers and children's postnatal passive smoking) was higher among children with a parental history of allergy (OR 1.49, 95\% CI 1.35-1.66) than in children without a parental history of allergy (OR 1.15, 95\% CI 1.00-1.32; p-value for interaction 0.043). Correspondingly, the odds ratio for exposure to maternal active smoking during pregnancy was increased in children with a parental history of allergy (OR 2.25, 95\% CI 1.99-2.54) compared to those without (OR 1.28, 95\% CI 1.09-1.50; p-value for interaction 0.011). Odds ratios were similar in males and females.

\section{Effects by region and cohort-specific effects}

When stratified by location, intra-European differences were noted, but the differences were not statistically significant. Northern-central European cohorts tended to have a higher risk of wheeze associated with passive maternal smoking during pregnancy (OR 1.36, 95\% CI 0.99-1.88) compared to southern-central European cohorts (OR 1.10, 95\% CI 0.96-1.26), but confidence intervals overlapped and the p-value for interaction was not significant $(\mathrm{p}=0.3))$. Similarly, odds ratios for active maternal smoking tended to be higher in northern European countries.

When conducting meta-analyses in order to evaluate cohort-specific effects, results obtained were nearly identical to those obtained by the pooled analyses (figs 1 and 2). An increased risk of wheezing in relation to passive smoking was observed for most cohorts, but there was significant heterogeneity between cohorts ( $\mathrm{p}$-value for heterogeneity 0.006). For active smoking, the pattern was more consistent with only one cohort not identifying an increased risk. We further examined heterogeneity by sequentially omitting each cohort (online supplementary table S3). The estimated risks for exposure to passive smoking among nonsmoking mothers when omitting one cohort at a time varied from 1.14 (95\% CI 1.00-1.28) when omitting ALSPAC to 1.18 (95\% CI 1.05-1.31) when omitting KOALA, compared to the overall risk of 1.16 (95\% CI 1.03-1.31) from the meta-analysis of all 15 cohorts. The corresponding estimated risks for active smoking varied from 1.58 (95\% CI 1.39-1.77) when omitting KOALA to 1.68 (95\% CI 1.4-1.89) when omitting PIAMA, compared to 1.65 (95\% CI 1.45-1.85) from the meta-analysis of all 15 cohorts.

Additional meta-analyses were applied using cohort-specific adjustment based on variables that have been previously associated with wheeze (e.g. existence of mould/dampness in the house, pet ownership, duration of breastfeeding and infant bedroom sharing), but these provided similar results to the main analysis that applied the same adjustment factors to all cohorts (data not shown). Analyses excluding the three cohorts (CONER, LEICESTER 1990 and RHEA) that had information on wheezing only for the first year of life (comprising $\sim 5 \%$ of the total population), gave very similar results to the overall analyses (data not shown). 


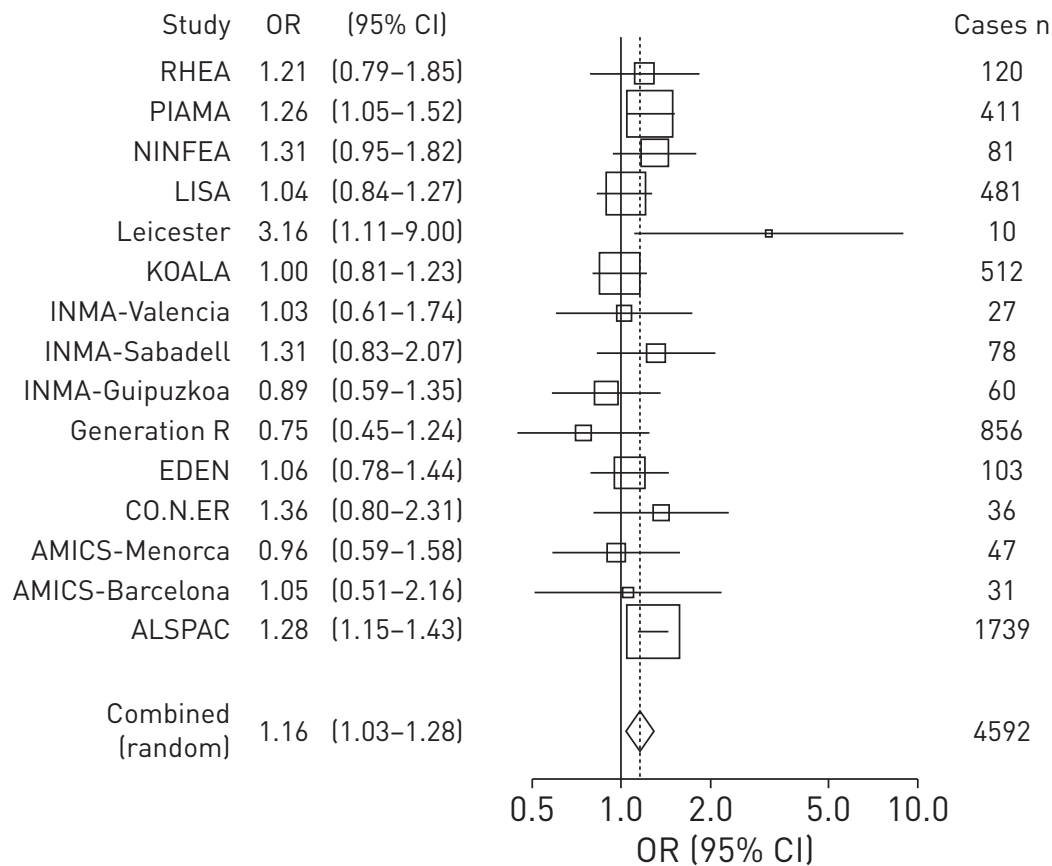

FIGURE 1 Associations between passive smoking in relation to wheeze up to 2 years of age in 15 European birth cohorts. Comparisons are for "nonsmoking mothers, mothers/infants exposed to SHS" (groups 2, 3 and 4 of table 2) and "nonsmoking mothers, mothers/infants not exposed to passive smoking" (group 1 in table 2). Cohort-specific odds ratios and $95 \%$ confidence intervals and meta-analysis using random effects, adjusted for sex of the child, siblings, birthweight, parental atopic status and gestational age.

\section{Discussion}

This large European Union-wide pooled analysis of cohort studies demonstrates that maternal passive smoking during pregnancy is an independent risk factor for wheeze in children in the first 2 years of life. Prenatal active smoking of the mother and postnatal passive smoking of the child pose additional threats to the child, with each timeframe of exposure adding an additional risk towards the development of wheeze before the age of 2 years. Notably, both prenatal maternal and postnatal infant passive smoke exposure were found to have an effect on the risk of developing wheeze, with a higher effect observed for prenatal maternal passive smoking than postnatal infant passive smoking; their combined exposure was associated with an even higher increase in risk. This effect was higher among children of parents with allergy than among children of parents without allergy.

Parental prenatal and postnatal active smoking has been shown to increase the risk for the development of asthma and wheeze later in life, a finding that has been previously identified by individual ENRIECO cohorts [12-15, 25-28]. A recent systematic review and meta-analysis of five cohorts from northern Europe and Japan identified that any prenatal maternal smoking was associated with an increase in risk of wheeze in children aged $\leqslant 2$ years (OR $1.41,95 \%$ CI 1.20-1.67) [12]. In this study, when we assessed the role of active maternal smoking during pregnancy alone (excluding any prenatal maternal passive smoking or postnatal infant passive smoke exposure) a lower risk (OR 1.33) was noted, although this estimate was based on small numbers. Combining active maternal prenatal smoking with passive smoking exposure led to higher risks for its combination with prenatal maternal passive smoking (OR 1.51), postnatal infant passive smoking (OR 1.74) and combined prenatal/postnatal exposure to passive smoking (OR 1.73). A potential explanation for the relatively modest although statistically significant risk associated with active smoking without passive smoking could be that this small group of mothers could be selected and perhaps quit smoking during pregnancy.

The significant shift that these analyses provide from the existing literature is the investigation between different timeframes of exposure on the risk of developing wheeze. Exposure to passive smoking alone was identified to increase the risk of developing wheeze if passive smoking exposure is persistent during the prenatal and postnatal period, while an increased risk for wheeze was noted among children who were only exposed to passive smoking prenatally. This latter risk of $\sim 10 \%$ could, in part, be due to residual confounding, but is still relevant for public health because exposure in many countries is very common. More importantly, as this study has shown, prenatal passive smoking exposure combined with other smoking exposures adds to the overall risk. These findings further stress the importance of protecting 


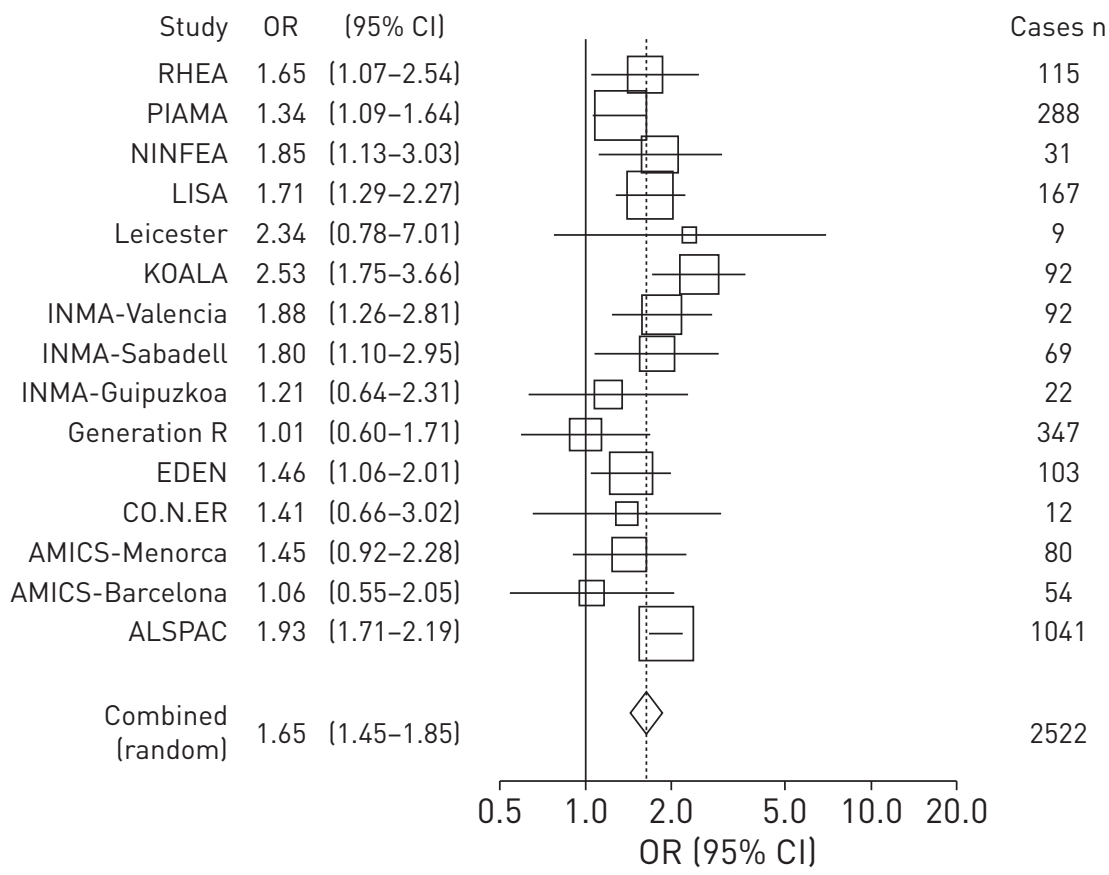

FIGURE 2 Associations between maternal active smoking during pregnancy in relation to wheeze up to 2 years of age in 15 European birth cohorts. Comparisons are for "active smoking mothers" (groups 5, 6, 7 and 8 of table 2) and "nonsmoking mothers, mothers/infants not exposed to passive smoking" (group 1 in table 2). Cohort-specific odds ratios and $95 \%$ confidence intervals and meta-analysis using random effects, adjusted for sex of the child, siblings, birthweight, parental atopic status and gestational age.

pregnant females and infants from passive smoke exposure, in both public and private areas [29]. Exposure to passive smoking in public places has been substantially regulated over the past decade with significant health gains for children [30,31]. While increased population awareness and the adoption of smoke-free environments have been found to reduce exposure inside the home [32], further action is needed to reduce exposure in other private areas that have been identified to contribute significantly to overall passive smoke exposure during the gestational period, such as exposure within the family car [33].

The association of smoking and wheeze may differ depending on familial history of allergy, although findings are inconsistent $[24,25,34,35]$. However, our stratified analysis indicated a substantial increase in the risk of developing wheeze among both those exposed to SHS and those who had a familial history of allergy and were exposed to prenatal active smoking. Furthermore, the possible higher risk of developing wheeze among northern European participants may be attributable to higher exposure to smoke indoors in the north compared to the south because of better ventilation of indoor air in the south due to warmer climate and having open windows more frequently. It could also be attributable to other contributing factors that predispose to the development of wheeze, such as the role of viral respiratory tract infections, which we could not investigate, or, less likely, to possible genetic variances between European populations.

Possible explanations for the increased risk of wheeze caused by passive tobacco smoke exposure in young children are proinflammatory mediator release, stimulation of inflammatory cells in the respiratory system, oxidative stress and increased cysteinyl leukotrienes [36, 37]. In addition, in recent years there has been an increase in the evidence for the effect of genetic and epigenetic mechanisms of action, through which maternal exposures may affect allergies and asthma in children [38-41].

One of the main strengths of the current analysis is the birth cohort study design of all studies included, which allowed us to assess temporal relationships between exposure to active and passive smoking and the development of wheeze. Although we did not have the exact timing of postnatal passive smoke in relation to timing of wheezing symptoms during the first 2 years it seems very unlikely that parents would start smoking in front of their children after, rather than before their children had a wheezing episode. Our large sample size provided a robust dataset that permitted the investigation of the independent role of each window of exposure (prenatal versus postnatal) and exposure type (active smoking versus passive) on the development of wheeze before the age of 2 years. To our knowledge our study is the first that has a sufficiently large sample size to separate the effects of different combinations of exposure. Conversely, the 
main limitation of our study was that we relied on parental report for both the exposure and the outcome, which may be subject to underreporting, recall and report bias. However, numerous studies have shown that participants (usually mothers) answer reliably about childhood exposure to SHS by their mother or their father [42-45]. In several studies effect estimates for respiratory symptoms or chronic diseases based on self-reported exposure to passive smoking are frequently equally strong, if not stronger, than those based on cotinine or other biomarkers [46]. Other limitations include the lack of information on other potential confounders (such as daycare attendance, social class and infections) and the inclusion only of studies participating in this large European project. Within our study, household and workplace passive smoking were used as a proxy for overall exposure to passive smoking, since a detailed evaluation for exposure in other locations was not available for several cohorts. It is possible that females who reported no passive smoking exposure at work or in their homes might have been exposed from other sources (i.e. cafes and bars); however, such misclassification of exposure is likely to have been nondifferential and thus would have been likely to attenuate the estimated associations rather than contribute to spurious links.

\section{Conclusion}

Our study suggests that maternal passive smoking exposure during pregnancy is an independent risk factor for wheeze in children up to the age of 2 years. The likelihood of developing wheeze during the first 2 years of life increased with exposure to both maternal prenatal active and maternal prenatal passive smoking and passive smoking of the child during the postnatal period; risks were higher in children of parents with allergy. These findings have public health implications and indicate the need for protecting pregnant females and young children from exposure to passive smoking by adopting smoke-free homes, work and other private and public places, as well as indicating the need for providing cessation opportunities to females and their partners.

\section{Acknowledgements}

We are grateful to all the families who took part in the studies and the study teams, which include midwives, interviewers, computer and laboratory technicians, clerical workers, research scientists, volunteers, managers, receptionists and nurses.

\section{References}

1 Oberg M, Jaakkola MS, Woodward A, et al. Worldwide burden of disease from exposure to second-hand smoke: a retrospective analysis of data from 192 countries. Lancet 2011; 377: 139-146.

2 US Department of Health and Human Services. The Health Consequences of Involuntary Exposure to Tobacco Smoke: a Report of the Surgeon General. Atlanta, US Department of Health and Human Services, Centers for Disease Control and Prevention, Coordinating Center for Health Promotion, National Center for Chronic Disease Prevention and Health Promotion, Office on Smoking and Health, 2006. www.surgeongeneral.gov/library/ secondhandsmoke/report/index.html Date last accessed: January 22, 2016. Last updated: 2006.

3 Herrmann M, King K, Weitzman M. Prenatal tobacco smoke and postnatal secondhand smoke exposure and child neurodevelopment. Curr Opin Pediatr 2008; 20: 184-190.

4 Wang L, Pinkerton KE. Detrimental effects of tobacco smoke exposure during development on postnatal lung function and asthma. Birth Defects Res C Embryo Today 2008; 84: 54-60.

5 Akinbami LJ, Schoendorf KV. Trends in childhood asthma: prevalence, health care utilization, and mortality Pediatrics 2002; 110: 315-322.

6 Jenkins MA, Clarke JR, Carlin JB, et al. Validation of questionnaire and bronchial hyperresponsiveness against respiratory physician assessment in the diagnosis of asthma. Int J Epidemiol 1996; 25: 609-616.

7 Hovland V, Riiser A, Mowinckel P, et al. The significance of early recurrent wheeze for asthma outcomes in late childhood. Eur Respir J 2013; 41: 838-845.

8 Henderson J, Granell R, Heron J, et al. Associations of wheezing phenotypes in the first 6 years of life with atopy, lung function and airway responsiveness in mid-childhood. Thorax 2008; 63: 974-980.

9 Savenije OE, Granell R, Caudri D, et al. Comparison of childhood wheezing phenotypes in 2 birth cohorts: ALSPAC and PIAMA. J Allergy Clin Immunol 2011; 127: 1505-1512.

10 Jackson DJ, Gangnon RE, Evans MD, et al. Wheezing rhinovirus illnesses in early life predict asthma development in high-risk children. Am J Respir Crit Care Med 2008; 178: 667-672.

11 Lodge CJ, Zaloumis S, Lowe AJ, et al. Early-life risk factors for childhood wheeze phenotypes in a high-risk birth cohort. J Pediatr 2014; 164: 289-294.

12 Burke H, Leonardi-Bee J, Hashim A, et al. Prenatal and passive smoke exposure and incidence of asthma and wheeze: systematic review and meta-analysis. Pediatrics 2012; 129: 735-744.

13 Pattenden S, Antova T, Neuberger M, et al. Parental smoking and children's respiratory health: independent effects of prenatal and postnatal exposure. Tob Control 2006; 15: 294-301.

14 Sonnenschein-van der Voort AM, de Kluizenaar Y, Jaddoe VW, et al. Air pollution, fetal and infant tobacco smoke exposure, and wheezing in preschool children: a population-based prospective birth cohort. Environ Health 2012; 11: 91.

15 Duijts L, Jaddoe VW, van der Valk RJ, et al. Fetal exposure to maternal and paternal smoking and the risks of wheezing in preschool children: the Generation R Study. Chest 2012; 141: 876-885.

16 Vrijheid M, Casas M, Bergström A, et al. European birth cohorts for environmental health research. Environ Health Perspect 2012; 120: 29-37.

17 Spratt M, Carpenter J, Sterne JA, et al. Strategies for multiple imputation in longitudinal studies. Am J Epidemiol 2010; 172: 478-487. 
18 Worldwide variation in prevalence of symptoms of asthma, allergic rhinoconjunctivitis, and atopic eczema: ISAAC. The International Study of Asthma and Allergies in Childhood (ISAAC) Steering Committee. Lancet 1998; 351: $1225-1232$

19 Eller E, Roll S, Chen CM, et al. Meta-analysis of determinants for pet ownership in 12 European birth cohorts on asthma and allergies: a GA2LEN initiative. Allergy 2008; 63: 1491-1498.

20 Antova T, Pattenden S, Brunekreef B, et al. Exposure to indoor mould and children's respiratory health in the PATY study. J Epidemiol Community Health 2008; 62: 708-714.

21 Martinez FD, Wright AL, Holberg CJ, et al. Maternal age as a risk factor for wheezing lower respiratory illnesses in the first year of life. Am J Epidemiol 1992; 136: 1258-1268.

22 Pirastu R, Bellu C, Greco P, et al. Indoor exposure to environmental tobacco smoke and dampness: respiratory symptoms in Sardinian children-DRIAS study. Environ Res 2009; 109: 59-65.

23 Tischer CG, Hohmann C, Thiering E, et al. Meta-analysis of mould and dampness exposure on asthma and allergy in eight European birth cohorts: an ENRIECO initiative. Allergy 2011; 66: 1570-1579.

24 Henderson AJ, Sherriff A, Northstone K, et al. Pre- and postnatal parental smoking and wheeze in infancy: cross cultural differences. Avon Study of Parents and Children (ALSPAC) Study Team, European Longitudinal Study of Pregnancy and Childhood (ELSPAC) Co-ordinating Centre. Eur Respir J 2001; 18: 323-329.

25 Håberg SE, Stigum H, Nystad W, et al. Effects of pre- and postnatal exposure to parental smoking on early childhood respiratory health. Am J Epidemiol 2007; 166: 679-686.

26 Strachan DP, Cook DG. Health effects of passive smoking. 6: Parental smoking and childhood asthma: longitudinal and case-control studies. Thorax 1998; 53: 204-212.

27 Neuman $\AA$, Hohmann C, Orsini N, et al. Maternal smoking in pregnancy and asthma in preschool children: a pooled analysis of eight birth cohorts. Am J Respir Crit Care Med 2012; 186: 1037-1043.

28 Fuentes-Leonarte V, Estarlich M, Ballester F, et al. Pre- and postnatal exposure to tobacco smoke and respiratory outcomes during the first year. Indoor Air 2015; 25: 4-12.

29 Kusel J, Timm B, Lockhart I. The impact of smoking in the home on the health outcomes of non-smoker occupants in the UK. Tob Induc Dis 2013; 11: 3.

30 Mackay D, Haw S, Ayres JG, et al. Smoke-free legislation and hospitalizations for childhood asthma. N Engl J Med 2010; 363: 1139-1145.

31 Been JV, Millett C, Lee JT, et al. Smoke-free legislation and childhood hospitalisations for respiratory tract infections. Eur Respir J 2015; 46: 697-706.

32 Akhtar PC, Haw SJ, Currie DB, et al. Smoking restrictions in the home and secondhand smoke exposure among primary schoolchildren before and after introduction of the Scottish smoke-free legislation. Tob Control 2009; 18: 409-415.

33 Vardavas CI, Fthenou E, Patelarou E, et al. Exposure to different sources of second-hand smoke during pregnancy and its effect on urinary cotinine and tobacco-specific nitrosamine (NNAL) concentrations. Tob Control 2013; 22: 194-200.

34 Keil T, Lau S, Roll S, et al. Maternal smoking increases risk of allergic sensitization and wheezing only in children with allergic predisposition: longitudinal analysis from birth to 10 years. Allergy 2009; 64: 445-451.

35 Vork KL, Broadwin RL, Blaisdell RJ. Developing asthma in childhood from exposure to secondhand tobacco smoke: insights from a meta-regression. Environ Health Perspect 2007; 115: 1394-1400.

36 Floreani AA, Rennard SI. The role of cigarette smoke in the pathogenesis of asthma and as a trigger for acute symptoms. Curr Opin Pulm Med 1999; 5: 38-46.

37 Peters JL, Boynton-Jarrett R, Sandel M. Prenatal environmental factors influencing IgE levels, atopy and early asthma. Curr Opin Allergy Clin Immunol 2013; 13: 187-192.

38 Kabesch M, Hoefler C, Carr D, et al. Glutathione S transferase deficiency and passive smoking increase childhood asthma. Thorax 2004; 59: 569-573.

39 Li H, Romieu I, Sienra-Monge JJ, et al. Genetic polymorphisms in arginase I and II and childhood asthma and atopy. J Allergy Clin Immunol 2006; 117: 119-126.

40 Meurs H, Maarsingh $\mathrm{H}$, Zaagsma J. Arginase and asthma: novel insights into nitric oxide homeostasis and airway hyperresponsiveness. Trends Pharmacol Sci 2003; 24: 450-455.

41 Herberth G, Bauer M, Gasch M, et al. Maternal and cord blood miR-223 expression associates with prenatal tobacco smoke exposure and low regulatory T-cell numbers. J Allergy Clin Immunol 2014; 133: 543-550.

42 Avila-Tang E, Elf JL, Cummings KM, et al. Assessing secondhand smoke exposure with reported measures. Tob Control 2013; 22: 156-163.

43 Brunekreef B, Leaderer BP, van Strien R, et al. Using nicotine measurements and parental reports to assess indoor air: the PIAMA birth cohort study. Prevention and Incidence of Asthma and Mite Allergy. Epidemiology 2000; 11 : $350-352$.

44 Gehring U, Leaderer BP, Heinrich J, et al. Comparison of parental reports of smoking and residential air nicotine concentrations in children. Occup Environ Med 2006; 63: 766-772.

45 Aurrekoetxea JJ, Murcia M, Rebagliato $\mathrm{M}$, et al. Determinants of self-reported smoking and misclassification during pregnancy, and analysis of optimal cut-off points for urinary cotinine: a cross-sectional study. BMJ Open 2013; 3: e002034.

46 Batty GD, Gale CR, Jefferis B, et al. Passive smoking assessed by salivary cotinine and self-report in relation to cause-specific mortality: 17-year follow-up of study participants in the UK Health and Lifestyle Survey. J Epidemiol Community Health 2014; 68: 1200-1203. 